\title{
Increased Prevalence of Ossification of Posterior Longitudinal Ligament and Increased Bone Mineral Density in Patients with Ossification of Nuchal Ligament
}

\author{
Ki-Wan Kim, Young-Min Oh, Jong-Pil Eun \\ Department of Neurosurgery, Chonbuk National University Medical School, Jeonju, Korea
}

\begin{abstract}
Objective: There are also few studies demonstrating the relationship between ossification of nuchal ligament (ONL) and ossification of posterior longitudinal ligament (OPLL). We compared the prevalence, location, and type of OPLL between patients with ONL and matched patients without ONL. We also compared the bone mineral densities (BMDs) between the 2 groups.

Methods: A total of 124 cervical ONL patients were enrolled in this study. The control group of 124 patients was matched with 124 patients with ONL by age and sex on a 1:1 basis to minimize confounding factors. We reviewed the prevalence, location, and type of OPLL in both groups.

Results: The prevalence of OPLL was almost 2.5 times greater in patients with ONL than those without ONL. The mean value of BMD in patients with ONL was greater at the lumbar spine (L1-L4) than in patients without ONL. The mean T score of the lumbar spine was $0.25 \pm 1.68$ in the patients with ONL and $-0.73 \pm 1.64$ in the patients without ONL.

Conclusion: The prevalence of OPLL in patients with ONL was significantly higher than in patients without ONL. Because ONL is innocuous and may be seen more readily than OPLL on simple cervical radiographs, clinicians should consider the possibility of coexisting OPLL when ONL, especially extensive ONL, is detected in patients with neck pain, radiculopathy, or myelopathy, to facilitate proper treatment.
\end{abstract}

Key Words: Ossification of posterior longitudinal ligament $\cdot$ Bone density $\cdot$ Spine $\cdot$ Nuchal ligament

\section{INTRODUCTION}

Various ligaments surround the vertebral column, including the anterior longitudinal ligament, posterior longitudinal ligament, ligamentum flavum, posterior ligament complex, and the nuchal ligament. These paravertebral ligaments may become ossified; conditions such as ossification of posterior longitudinal ligament (OPLL), diffuse idiopathic skeletal hyperostosis (DISH), and ossification of ligamentum flavum (OLF) can lead to neurological symptoms due to rigidity and compression of the spinal cord and nerve roots. Many studies have investigated the different forms of paravertebral ligamentous ossification.

The nuchal ligament is a triangular fibrous membrane, which extends from the external occipital protuberance to the spi-

- Received: August 11, 2016 • Revised: September 5, 2016

- Accepted: September 13, 2016

Corresponding Author: Young-Min Oh

Department of Neurosurgery, Chonbuk National University Hospital, Chonbuk National University Medical School, 20 Geonji-ro, Deokjin-gu, Jeonju 54907, Korea

Tel: +82-63-250-1870, Fax: +82-63-277-3273

E-mail: timoh@jbnu.ac.kr

@This is an open access article distributed under the terms of the Creative Commons Attribution Non-Commercial License (http://creativecommons.org/licenses/by-nc/4.0/) which permits unrestricted non-commercial use, distribution, and reproduction in any medium, provided the original work is properly cited. nous process of the 7 th cervical vertebra ${ }^{4,12}$. It maintains the lordotic alignment and limits the movements of cervical spine $^{6,17)}$. Ossification of nuchal ligament $(\mathrm{ONL})$ is identified as a radio-opaque formation in the soft tissues behind the spinous processes of the cervical spine. It is usually innocuous, and is more often observed in Asian patients. Trauma, chronic strain in the cervical spinal ligaments, age, and systemic conditions might cause ONL ${ }^{19,20)}$. Many authors have proposed that ONL may be classified as a spinal ligament ossification syndrome, similar to OPLL, DISH, or OLF ${ }^{13,20)}$. Because of its similarities to and links with the ossification of other spinal ligaments, it is thought that ONL may coexist as part of a disorder with, or be a risk factor for, other cervical degenerative diseases. However, unlike the ossification of other spinal ligaments, there is a lack of studies about the characteristics and the clinical significance of ONL. The effect of ONL on patients' symptoms and cervical function is still unclear. There are also few studies demonstrating the relationship between ONL and OPLL. We compared the prevalence, location, and type of OPLL between patients with ONL and matched patients without ONL. We also compared the bone mineral densities (BMDs) between the 2 groups.

\section{MATERIALS AND METHODS}

Medical records of patients in our Department of Neuro- 
surgery who had been diagnosed with cervical ONL from January 2010 to December 2015 were reviewed. Cervical ONL was diagnosed by plain cervical lateral radiography and computed tomography (CT). We included patients with cervical degenerative diseases such as cervical disc disease, cervical spondylosis, or cervical OPLL, and excluded patients with nondegenerative cervical diseases such as trauma, infection, deformity, or inflammation; and patients with previous cervical procedures. A total of 124 cervical ONL patients were enrolled in this study. The study also included a control group, which consisted of patients with cervical degenerative disease who visited the Department of Neurosurgery during the same study period. Patients with ONL were excluded from the control group, but patients with cervical spondylosis and cervical disc disorder were included. Patients who underwent decompressive posterior laminectomy were also excluded. The control group of 124 patients was matched with 124 patients with ONL by age and sex on a 1:1 basis to minimize confounding factors. We reviewed the prevalence, location, and type of OPLL in both groups. Institutional Review Board of Chonbuk National University Hospital approved this study (CUH 2013-09-004-007).

ONL was diagnosed through cervical X-ray and CT imaging, and subdivided into round, rod, and segmental types ac-

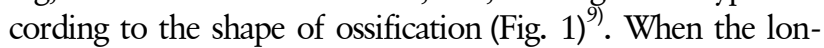
gest axis of an ONL was less than $10 \mathrm{~mm}$ in the sagittal plane, it was classified as round; when exceeding $10 \mathrm{~mm}$ in the sagittal plane, it was classified as a rod type ONL if continuous or a segmented type ONL if discontinuous.

Cervical OPLL was diagnosed with cervical CT, based on
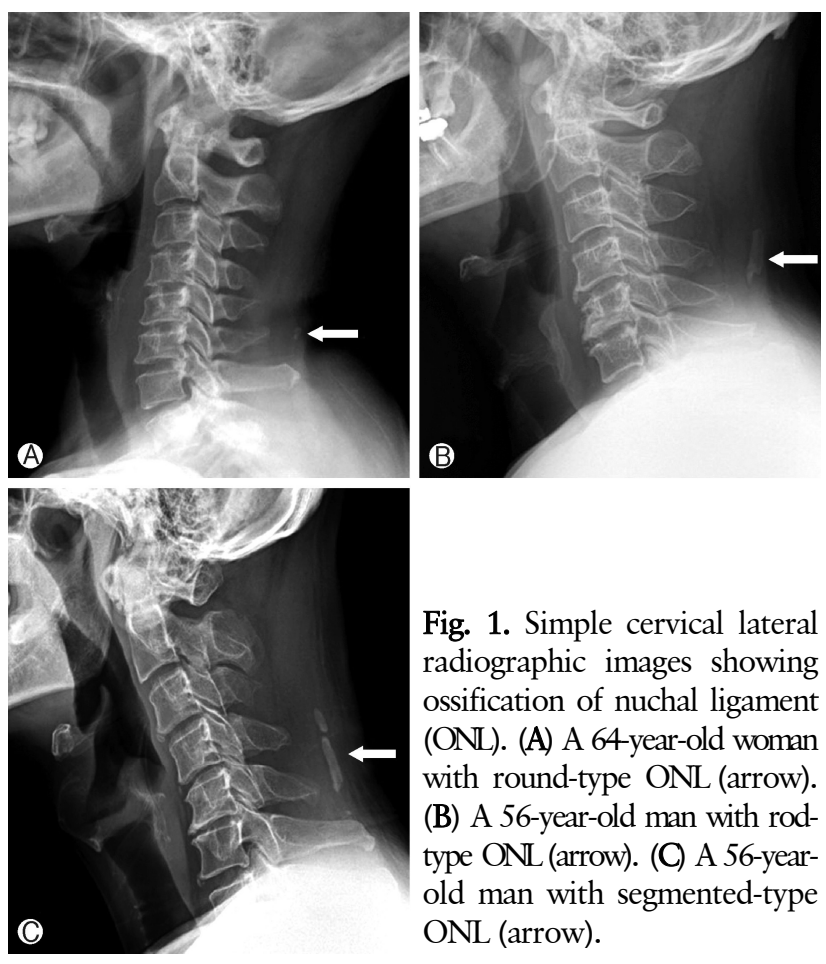

Fig. 1. Simple cervical lateral radiographic images showing ossification of nuchal ligament (ONL). (A) A 64-year-old woman with round-type ONL (arrow). (B) A 56-year-old man with rodtype ONL (arrow). (C) A 56-yearold man with segmented-type ONL (arrow). previously determined criteria. The extent of cervical OPLL involvement was determined by the number of cervical spine levels with OPLL. This ranged from 1 to 6 levels; patients were divided into 2 groups, a short level (1-3 levels) OPLL group and a long level (4-6 levels) OPLL group.

$\mathrm{BMD}$ was measured at the lumbar spine (L1-L4) using dual-energy X-ray absorptiometry apparatus (GE Lunar, Madison, WI, USA). BMD of the lumbar spine was measured in the standard anterior-posterior projection. Results are given as $\operatorname{BMD}\left(\mathrm{g} / \mathrm{cm}^{2}\right)$ and T scores. T scores were calculated by taking the differences between the measured BMD and the mean $\mathrm{BMD}$ of healthy young adults matched for sex and ethnicity, divided by the SD of the young adult population. T scores less than -2.5 indicate osteoporosis ${ }^{8)}$.

Baseline characteristics and differences in BMD were analyzed using the paired t-test. We used the chi-square test for comparing the prevalence of OPLL in patients with ONL and in patients without ONL. The relationship between the number of OPLL levels and types of ONL were also investigated with chi-square test. For all statistical analyses, SPSS version 18.0 (SPSS Inc., Chicago, IL, USA) was used, and statistical significance was defined as $\mathrm{p}<0.05$.

\section{RESULTS}

The mean age of the patients was 57.8 years (range, 38-79 years), and there were 196 male patients and 52 female patients (Table 1). Among the patients with ONL, 49 patients showed round type and 50 patients showed rod type; the others (25 patients) showed segmented type (Table 2). ONL was most frequent in the sixth decade (50 patients, $40.4 \%$ ) (Table 2).

Table 1. Demographics of the patients and comparison between patients with ONL and patients without ONL

\begin{tabular}{lccc}
\hline \hline \multicolumn{1}{c}{ Variable } & $\begin{array}{l}\text { Patients with } \\
\text { ONL }(\mathrm{n}=124)\end{array}$ & $\begin{array}{c}\text { Patients without } \\
\text { ONL }(\mathrm{n}=124)\end{array}$ & p-value \\
\hline Age (yr) & 57.8 & 57.8 & \\
$\begin{array}{l}\text { Sex, male:female } \\
\text { OPLL }\end{array}$ & $98: 26$ & $98: 26$ & \\
$\quad(+)$ & & & $<0.001^{*}$ \\
$\quad(-)$ & $70(56.5)$ & $28(22.6)$ & \\
Bone mineral & $0.25 \pm 1.68$ & $-0.73 \pm 1.64$ & $<0.001^{+}$ \\
density & & & \\
Total & $124(100)$ & $124(100)$ & \\
\hline
\end{tabular}

Values are presented as number, number (\%), or mean \pm standard deviation.

ONL, ossification of nuchal ligament; OPLL, ossification of posterior longitudinal ligament.

"Chi-square test. ${ }^{\dagger}$ t-test. 


\section{Comparison of Prevalence of OPLL between Patients with ONL and Patients without ONL}

In the patients with ONL, 70 patients (56.5\%) were identified as having OPLL on radiographs, while in the patients without ONL, only 28 patients (22.6\%) were identified as having OPLL. The prevalence of OPLL was almost 2.5 times greater in patients with ONL than those without ONL. The relative risk was 2.5 and odds ratio was 4.444 (95\% confidence interval, 2.563-7.708).

\section{Association between Type of ONL and Extent of OPLL}

We analyzed the extent of OPLL in accordance with the type of ONL. Patients with rod type and segmented type ONL showed a higher incidence of long level OPLL than the patients with round type ONL, which was statistically significant $(\mathrm{p}<0.000867)$. Among the patients with round type ONL, 6 of $28(21.4 \%)$ showed long level OPLL, while among the patients with rod and segmented type ONL, 26 of 42 (61.9\%) showed long level OPLL (Table 3).

\section{Differences in BMD between Patients with ONL and Patients without ONL}

There was a difference in the mean values of BMD between

Table 2. The prevalence of ossification of nuchal ligament according to the age

\begin{tabular}{|c|c|c|c|c|}
\hline \multirow{2}{*}{ Age (yr) } & \multicolumn{3}{|c|}{ Type } & \multirow{2}{*}{ Total, n (\%) } \\
\hline & Round & Rod & Segmented & \\
\hline$<40$ & 0 & 2 & 0 & $2(1.6)$ \\
\hline $40-49$ & 7 & 9 & 4 & $20(16.1)$ \\
\hline $50-59$ & 23 & 17 & 11 & $50(40.4)$ \\
\hline $60-69$ & 9 & 19 & 8 & $37(29.8)$ \\
\hline$>69$ & 10 & 3 & 2 & 15 (12.1) \\
\hline Total, n (\%) & 49 (39.5) & $50(40.3)$ & $25(20.2)$ & $124(100)$ \\
\hline
\end{tabular}

Table 3. Association between type of ONL and extent of OPLL

\begin{tabular}{|c|c|c|c|}
\hline Extent of OPLL & $\begin{array}{c}\text { Round type } \\
\text { ONL }\end{array}$ & $\begin{array}{c}\text { Rod \& } \\
\text { segmented } \\
\text { type ONL }\end{array}$ & p-value" \\
\hline Short level (1-3 levels) & $22(78.6)$ & $16(38.1)$ & \\
\hline Long level (4-6 levels) & $6(21.4)$ & $26(61.9)$ & \\
\hline Total & $28(100)$ & $42(100)$ & 0.000867 \\
\hline
\end{tabular}

patients with ONL and those without. The mean value of $\mathrm{BMD}$ in patients with ONL was greater at the lumbar spine (L1-L4) than in patients without ONL. The mean T score of the lumbar spine was $0.25 \pm 1.68$ in the patients with ONL and $-0.73 \pm 1.64$ in the patients without ONL (Table 1).

\section{DISCUSSION}

ONL is usually asymptomatic and may be detected incidentally on cervical radiographs. In 1963, Scapinelli ${ }^{19)}$ reported that ONL typically occurred after the third decade of life, with maximal frequency in the sixth decade, and that the overall incidence was $11.3 \%$ in men and $3.5 \%$ in women. They proposed that trauma or stress in the cervical spine plays a role in the development of ONL, as ONL appears at the levels of the cervical spine where mobility is greatest.

In concordance with previous studies, ONL was most frequent in the sixth decade in the present study (Table 2), and the incidence of ONL was higher in men than in women and increased with age. ONL appeared most frequently at the C5 vertebra, confirming its occurrence at the level of greatest mobility. Because men tend to be more physically active than women, it is suggested that chronic overload of the cervical spine with age and cervical motion may promote ONL, as reported in previous studies ${ }^{19)}$.

Recently, some investigators have proposed that ONL may be a type of spinal ligament ossification syndrome ${ }^{13,18,20)}$. Spinal ligament ossification syndrome, which includes OPLL, OLF, and DISH, is a multifactorial disease with complex and multiple interactions between genetic and environmental factors ${ }_{16,22,24)}$. Previous reports have identified the causative associations of DISH, OPLL, and OLF ${ }^{2,15)}$. Calcium-regulating hormones, glucose metabolism hormones, and growth factors such as bone morphogenic hormones and tumor growth factor $\beta_{1}$ are thought to be important in the pathogenesis of the ossification of the spinal ligament, although the underlying mechanisms remain uncertain ${ }^{10}$.

OPLL is characterized by growth of the posterior longitudinal ligament with the development of ossification centers. It is common in Asia, and the prevalence of cervical OPLL is reported to range from $0.6 \%$ to $2.2 \%$ in the Korean populations ${ }^{7}$. The underlying cause of OPLL is still unclear and is believed to be influenced by several genetic and hormonal factors ${ }^{5,25}$. There are few studies demonstrating the relationship between OPLL and ONL. In 1996, Shingyouchi et al. ${ }^{20)}$ reported that ossification of anterior longitudinal ligament, ONL, and OPLL had etiologic similarities in terms of age, sex, and obesity; however, ONL had additional risk factors because ONL is more related to dynamic stress. In this study, the prevalence of OPLL in patients with ONL was significantly higher than in patients without ONL (56.5\% vs. $22.6 \%)$. This suggests that the development of ONL is closely related to the development of OPLL. Because ONL can be easily detected with cervical radiographs, clinicians should consider the possibility of OPLL when ONL is detected. 
In the present study, we classified ONL into round, rod, and segmented types. Previously, An et al. ${ }^{1)}$ studied $240 \mathrm{pa-}$ tients with ONL using this classification. Among their cases, the rod type was observed in $39.5 \%$, the round type in $40.3 \%$, and the segmented type in $20.2 \%$ of patients. In the third decade of life, round-type ONL was the most common, whereas rod type was the most common after the fourth decade of life. The authors reported that degenerative changes with aging might play a role in the development and progression of ONL, and suggested that round-type ONL develops initially and grows into the rod type and the segmented type due to degenerative changes and aging. Also, in the present study, the short-level OPLL patients (one to three vertebrae) showed a high incidence of round-type ONL, and the long-level OPLL patients (4 to 6 vertebrae) showed a high incidence of the rod and segmented types. This observation suggests that the same pathogenesis may promote the progression of OPLL and the development of ONL from the round to the rod and segmented types and that ONL may be associated with other spinal ligament ossification syndromes, such as DISH and OLF. Wang et al. ${ }^{23)}$ reported that the incidence of ONL was almost 2 times greater in patients with OPLL than in other spondylosis patients; and that sex, aging, and OPLL were related with the formation of ONL, which was concordant with our results. Generally, somatic conditions may lead to ossification of spinal ligaments ${ }^{20)}$, and patients with OPLL commonly showed concomitant ossification of other ligaments in the cervical spine ${ }^{14)}$. The systemic factors related to OPLL pathogenesis may promote the development and progression of ONL; this link needs to be demonstrated through further study.

Interestingly, in the present study, BMDs were higher in ONL patients than in the control group. Several studies have investigated bone and mineral metabolism in patients with OPLL. Matsui et al. ${ }^{11)}$ demonstrated increased levels of serum procollagen type I carboxyl-terminal peptide and intact osteocalcin in patients with OPLL. Goto et al. ${ }^{3)}$ reported that insulin-like growth factor-I induces histological changes and elevation of alkaline phosphatase activity in OPLL cell lines much more than in non-OPLL cells. Recently, through a case-control study, Sohn and Chung ${ }^{21)}$ suggested that BMD increases systemically and the prevalence of osteoporosis decreases in cervical OPLL patients. In our study, BMDs increased in ONL patients and OPLL patients alike. This may indicate that there are close similarities in bone and mineral metabolism between OPLL and ONL.

There are several limitation of this study. Because ONL patients were accumulated retrospectively, there could be selection bias, and other potential confounding factors, such as occupation, physical activity, smoking, and diet, was not controlled. Also, we did not measure hormonal level and bone turnover markers. Since OPLL prevalence and BMD are increased in the ONL group than the control group, there could be a difference in hormone and bone turnover markers. To clarify this, further prospective studies regarding the relationships between ONL and systemic hormones should be needed.

\section{CONCLUSION}

The prevalence of OPLL in patients with ONL was significantly higher than in patients without ONL. Morphologic development of ONL was related to the degree of OPLL involved. This indicates that the formation of ONL is related to OPLL. However, the relationships between ONL and OPLL still require further study. Because ONL is innocuous and may be seen more readily than OPLL on simple cervical radiographs, clinicians should consider the possibility of coexisting OPLL when ONL, especially extensive ONL, is detected in patients with neck pain, radiculopathy, or myelopathy, to facilitate proper treatment. As the BMDs of patients with ONL were increased, we suggest that patients with ONL have little risk of suffering from osteoporosis.

\section{CONFLICT OF INTEREST}

No potential conflict of interest relevant to this article was reported

\section{ACKNOWLEDGMENTS}

This paper was supported by Fund of Biomedical Research Institute, Chonbuk National University Hospital .

\section{REFERENCES}

1. An $\mathrm{CH}$ : Study on the nuchal ligament ossification on lateral cephalometric radiograph. Korean J Oral Maxillofac Radiol 39: 7-11, 2009

2. Ehara S, Shimamura T, Nakamura R, Yamazaki K: Paravertebral ligamentous ossification: DISH, OPLL and OLF. Eur J Radiol 27:196-205, 1998

3. Goto K, Yamazaki M, Tagawa M, Goto S, Kon T, Moriya H, et al: Involvement of insulin-like growth factor I in development of ossification of the posterior longitudinal ligament of the spine. Calcif Tissue Int 62:158-165, 1998

4. Johnson GM, Zhang M, Jones DG: The fine connective tissue architecture of the human ligamentum nuchae. Spine (Phila Pa 1976) 25:5-9, 2000

5. Jun JK, Kim SM: Association study of fibroblast growth factor 2 and fibroblast growth factor receptors gene polymorphism in korean ossification of the posterior longitudinal ligament patients. J Korean Neurosurg Soc 52:7-13, 2012

6. Kadri PA, Al-Mefty O: Anatomy of the nuchal ligament and its surgical applications. Neurosurgery 61(5 Suppl 2):301-304, 2007

7. Kang MS, Lee JW, Zhang HY, Cho YE, Park YM: Diagnosis of cervical OPLL in lateral radiograph and MRI: is it Reliable? Korean J Spine 9:205-208, 2012

8. Kanis JA, Melton LJ 3rd, Christiansen C, Johnston CC, Khaltaev N: The diagnosis of osteoporosis. J Bone Miner Res 9:11371141, 1994 
9. Kim DG, Oh YM, Eun JP: The clinical significance of ossification of ligamentum nuchae in simple lateral radiograph: a correlation with cervical ossification of posterior longitudinal ligament. J Korean Neurosurg Soc 58:442-447, 2015

10. Luo J, Wei X, Li JJ: Clinical significance of nuchal ligament calcification and the discussion on biomechanics. Zhongguo Gu Shang 23:305-307, 2010

11. Matsui H, Yudoh K, Tsuji H: Significance of serum levels of type I procollagen peptide and intact osteocalcin and bone mineral density in patients with ossification of the posterior longitudinal ligaments. Calcif Tissue Int 59:397-400, 1996

12. Mercer SR, Bogduk N: Clinical anatomy of ligamentum nuchae. Clin Anat 16:484-493, 2003

13. Mine T, Kawai S: Ultrastructural observations on the ossification of the supraspinous ligament. Spine (Phila Pa 1976) 20:297-302, 1995

14. Ohtsuka K, Terayama K, Yanagihara M, Wada K, Kasuga K, Machida T, et al: A radiological population study on the ossification of the posterior longitudinal ligament in the spine. Arch Orthop Trauma Surg 106:89-93, 1987

15. Ono M, Russell WJ, Kudo S, Kuroiwa Y, Takamori M, Motomura S, et al: Ossification of the thoracic posterior longitudinal ligament in a fixed population. Radiological and neurological manifestations. Radiology 143:469-474, 1982

16. Sakou T, Taketomi E, Matsunaga S, Yamaguchi M, Sonoda S, Yashiki S: Genetic study of ossification of the posterior longitudinal ligament in the cervical spine with human leukocyte antigen haplotype. Spine (Phila Pa 1976) 16:1249-1252, 1991

17. Sasai K, Saito T, Akagi S, Kato I, Ogawa R: Cervical curvature after laminoplasty for spondylotic myelopathy: involvement of yellow ligament, semispinalis cervicis muscle, and nuchal ligament. J Spinal Disord 13:26-30, 2000

18. Scapinelli R: Localized ossifications in the supraspinous and interspinous ligaments of adult man. Rays 13:29-33, 1988

19. Scapinelli R: Sesamoid bones in the ligamentum nuchae of man. J Anat 97:417-422, 1963

20. Shingyouchi Y, Nagahama A, Niida M: Ligamentous ossification of the cervical spine in the late middle-aged Japanese men. Its relation to body mass index and glucose metabolism. Spine (Phila Pa 1976) 21:2474-2478, 1996

21. Sohn S, Chung CK: Increased bone mineral density and decreased prevalence of osteoporosis in cervical ossification of the posterior longitudinal ligament: a case-control study. Calcif Tissue Int 92:28-34, 2013

22. Terayama K: Genetic studies on ossification of the posterior longitudinal ligament of the spine. Spine (Phila Pa 1976) 14: 1184-1191, 1989

23. Wang H, Zou F, Jiang J, Lu F, Chen W, Ma X, et al: Analysis of radiography findings of ossification of nuchal ligament of cervical spine in patients with cervical spondylosis. Spine (Phila Pa 1976) 39:E7-11, 2014

24. Yamaguchi M: Genetic study on OPLL in the cervical spine with HLA haplotype. Nihon Seikeigeka Gakkai Zasshi 65:527535, 1991

25. Yanagihara M: An epidemiological study on the ossification of the anterior longitudinal ligament of the spine. Nihon Seikeigeka Gakkai Zasshi 60:187-202, 1986 